\title{
Venous Thromboembolism While on Anticoagulation With Apixaban
}

\author{
Farah Mazahreh ${ }^{1}$, Fuad Habash ${ }^{1}$, Angel López-Candales ${ }^{2}$ \\ 1. Cardiology, University of Arkansas for Medical Sciences, Little Rock, USA 2. Cardiovascular Medicine, University of \\ Missouri Kansas City, Kansas City, USA
}

Corresponding author: Farah Mazahreh, fmazahreh@uams.edu

\begin{abstract}
Venous thromboembolism (VTE) is a common condition whose pathophysiology is explained by Virchow's triad with stasis, hypercoagulability, and endothelial injury. Direct oral anticoagulants (DOACs) showed non-inferiority when compared with conventional treatment using subcutaneous low molecular weight heparin (LMWH) and warfarin, but treatment failure is a concern and remains a challenge for physicians. In our case report, we present a patient who had VTE in the form of a saddle pulmonary embolus while on apixaban.
\end{abstract}

Categories: Cardiology, Hematology

Keywords: cardiovascular diseases / blood, venous thromboembolism (vte), saddle pulmonary embolism, failed anticoagulation

\section{Introduction}

Venous thromboembolic disease occurs when thrombus develops within the deep veins, followed by propagation and dislodgment of the thrombus causing a pulmonary embolism, which occurs in one-third of patients with deep vein thrombosis (DVT) [1]. The high mortality rate of pulmonary embolism (PE) even among young adults [2] and the possibility of developing acute right ventricular failure makes clinical suspicion, early diagnosis, and management of paramount importance. Current guidelines for the prevention of PE in those with a previous history of DVT expanded to include treatment with direct oral anticoagulants (DOACs) such as apixaban (Anti-factor Xa) [3]. Although major trials like Apixaban for the Initial Management of Pulmonary Embolism and Deep-Vein Thrombosis as First-Line Therapy (AMPLIFY) [4] and RECOVER ${ }^{\mathrm{TM}}$ II [5] for apixaban and dabigatran, respectively, confirmed the efficacy of these medications with significant advantages over conventional treatment, it is still to be known if cases with treatment failure warrant further investigation of the safety and efficacy of the newer medications.

Review began 03/17/2021 Review ended 05/23/2021 Published 05/23/2021

\section{๑) Copyright 2021}

Mazahreh et al. This is an open access article distributed under the terms of the Creative Commons Attribution License CC-BY 4.0., which permits unrestricted use, distribution, and reproduction in any medium, provided the original author and source are credited.

\section{Case Presentation}

A 73-year-old male patient with diabetes, hypertension, hyperlipidemia, and a history of unprovoked deep venous thrombosis on apixaban $5 \mathrm{mg}$ twice daily, with no evidence of DVT resolution at that time, presented to the hospital with shortness of breath for three days. The initial episode of shortness of breath was associated with chest pain and diaphoresis that lasted for 30 minutes. The patient was compliant with his medication and active at home. He had no family history of recurrent thrombosis.

On physical examination, the patient was in mild respiratory distress. Vital signs showed a heart rate of 95 bpm, respiratory rate of 20/minute, and oxygen saturation of $87 \%$ on room air. Cardiac examination showed normal first (S1) and second (S2) heart sounds, no added abnormal cardiac sounds, and, in particular, there was no appreciable splitting of S2. The respiratory examination was unremarkable.

Brain natriuretic peptide (BNP) was elevated (358.6 pg/ml; Ref: $</=100 \mathrm{pg} / \mathrm{mL}$ ), initial troponin I was elevated (0.114 ng/ml; Ref 0.0-.028 ng/ml), and lactic acid was elevated ( $3.8 \mathrm{mmol} / \mathrm{L}$; Ref $0.5-2 \mathrm{mmol} / \mathrm{L}$ ). International normalized ratio (INR), activated partial thromboplastin time (aPTT), platelet count, renal function, and electrolytes were within the normal range. Electrocardiography (ECG) was significant for Twave inversions in anterior leads (V1-3), with a rightward shift of the QRS axis but otherwise no ST-segment deviation. Coronavirus disease 2019 (COVID-19) testing using nucleic acid amplification test (NAAT) for severe acute respiratory syndrome coronavirus 2 (SARS-CoV-2) was negative. Chest X-ray (Figure 1 ) showed increased cardiac silhouette, small left pleural effusion, and no evidence of pulmonary consolidation. 


\section{Cureus}

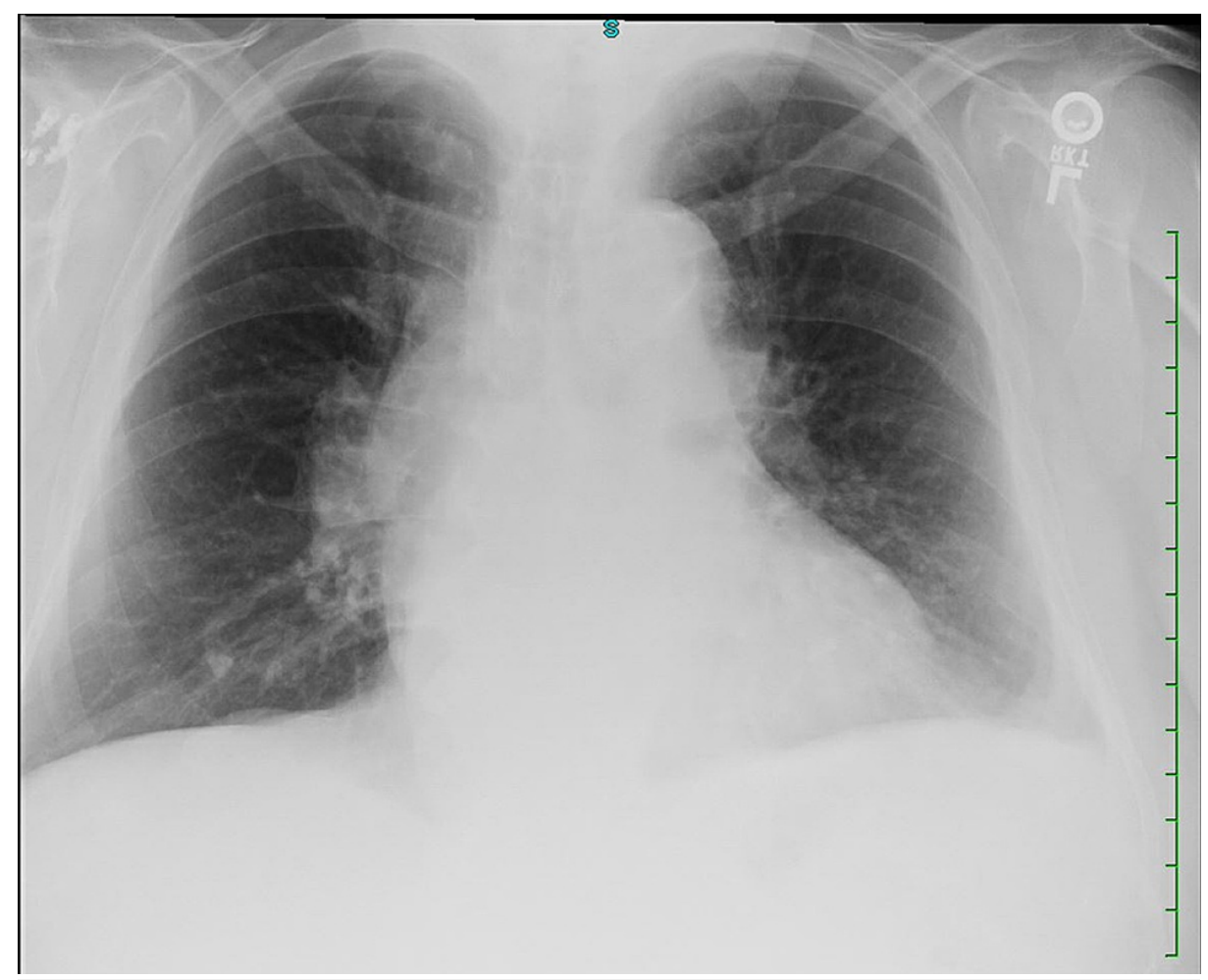

\section{FIGURE 1: Chest X-ray showing borderline cardiac silhouette with a small left pulmonary effusion but without pulmonary infiltrates or consolidation}

Myocardial ischemia was on the differential diagnosis list, with ECG findings concerning for acute coronary syndrome (ACS), including T-wave inversion and ST-segment depression, which may well be PE masquerading as myocardial infarction (MI). Considering the patient's presentation, ECG findings (Figure 2) of Wellens type B (deeply inverted T-waves in V2-V3, which is $89 \%$ specific for significant stenosis of the left anterior descending artery) [6] and a previous history of coronary calcification on a previous lung CT scan, ACS had to be excluded and cardiology was consulted for a possible percutaneous coronary angiogram (PCI).

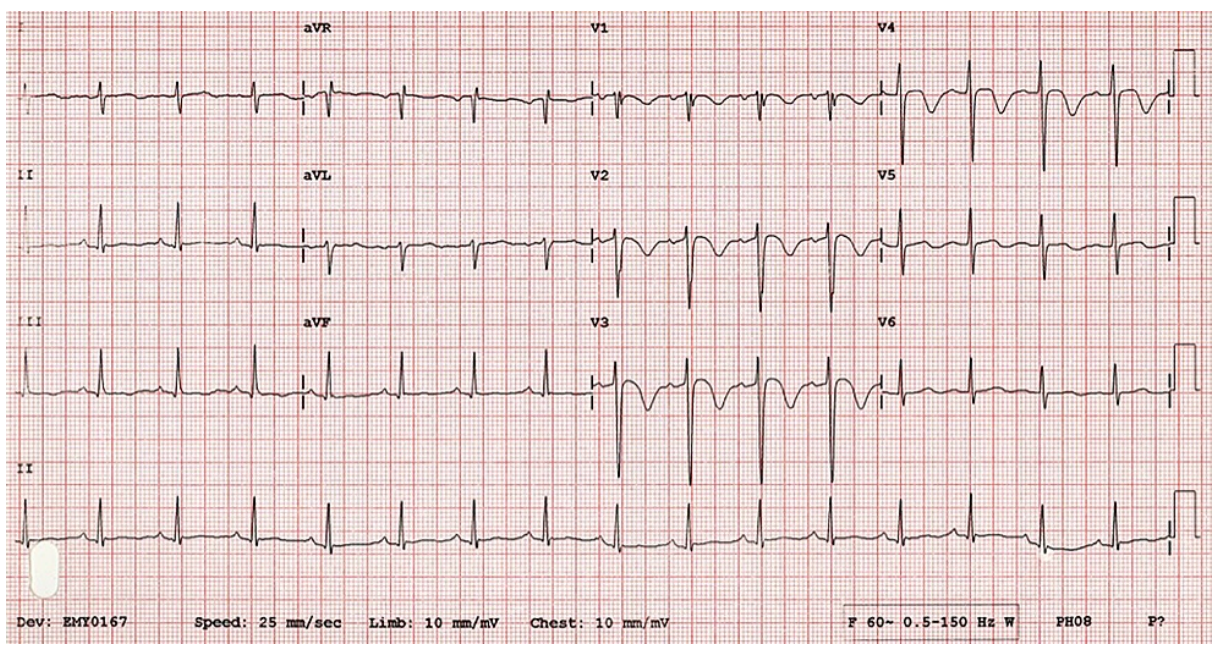

FIGURE 2: Electrocardiogram: type B Wellens

In the meantime, an initial bedside echocardiogram was technically difficult due to the patient's body habitus but revealed right ventricle dilation with right ventricular strain pattern and mild global systolic dysfunction (ejection fraction of $45 \%-50 \%$ ). With the current echocardiographic findings, pulmonary embolus was high on our differential. The CT-pulmonary embolus protocol with contrast showed a large saddle embolus with bilateral occlusive and non-occlusive emboli in both the right and left pulmonary 


\section{Cureus}

arteries (Figure 3). A formal echocardiogram was negative for both regional wall abnormalities and patent foramen oval with agitated saline.

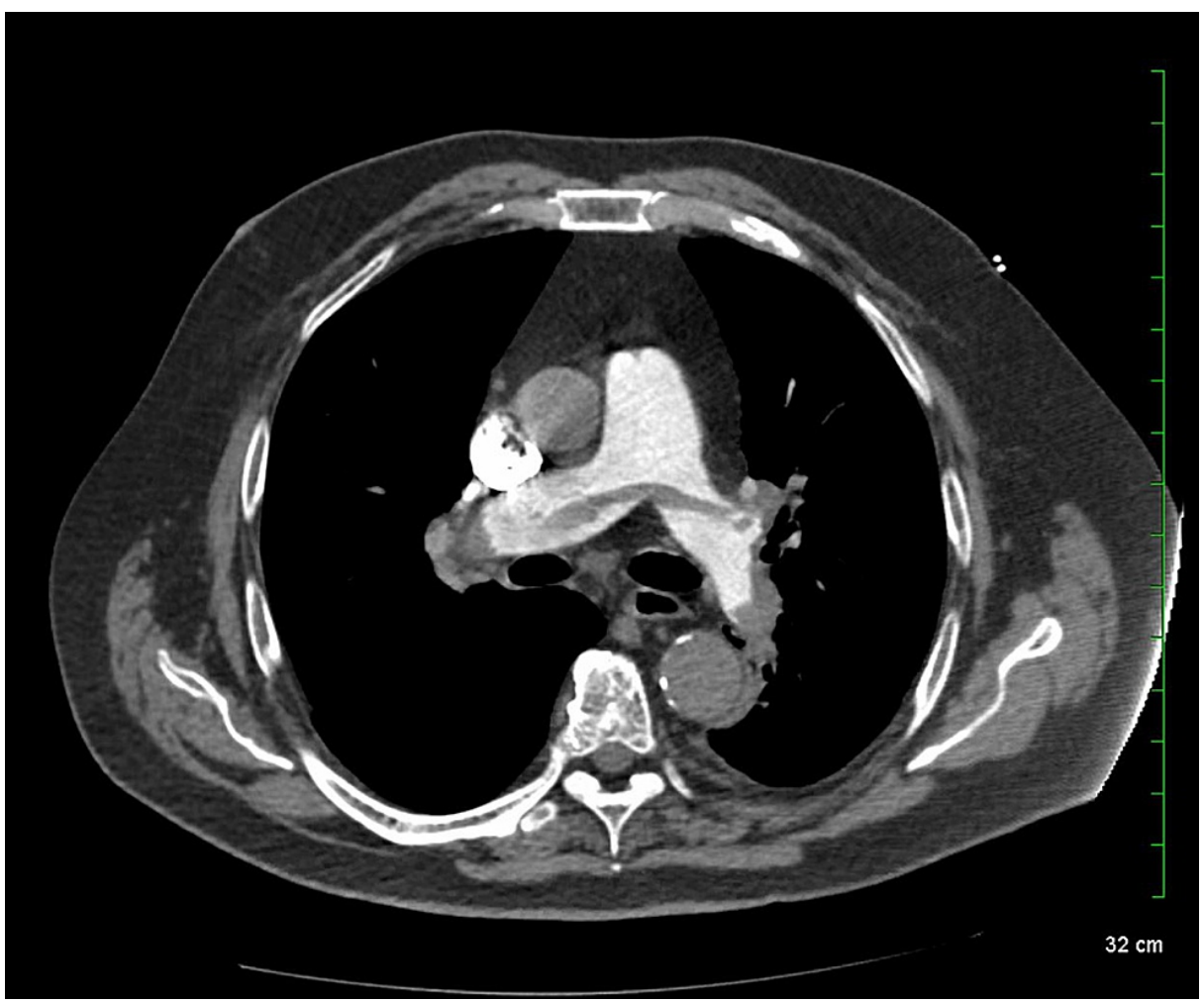

\section{FIGURE 3: CT-pulmonary embolus protocol: saddle embolus with bilateral occlusive and non-occlusive emboli in both the right and left branches of the pulmonary artery}

Patient's management included supplemental oxygen, initiation of un-fractionated heparin infusion as per protocol, and admission to the intensive care unit. CT chest, abdomen, and pelvis showed no evidence of malignancy, and hyper-coagulability testing was negative as well.

\section{Discussion}

Pulmonary embolism leads to a partial or complete blockage of pulmonary artery branches, most commonly due to embolization of a leg deep venous thrombus [1]. Pulmonary embolus is the third most common cause of cardiovascular death [7].

PE can be classified into massive (hemodynamic instability), sub-massive (right ventricular dysfunction on echocardiogram), and non-massive (without hemodynamic instability or right ventricular dysfunction). Diagnosis can be made by CT-PE showing filling defects in the pulmonary artery and its branches.

Management depends on classification; it was shown that systemic thrombolytic therapy improves survival in patients with massive PE. It can also be used in sub-massive PE but there is a considerable risk of bleeding. In addition, it was proven that the mortality rate in those treated with heparin anticoagulation is less than $3 \%$, which makes it the treatment of choice in sub-massive PE [8].

Ekosonic Endovascular System (EKOS) was then introduced, potentially reducing the risk of bleeding. It is catheter-based pharmacochemical thrombolysis that employs high-frequency ultrasound to augment the penetration of thrombolytics into selective targets [9]. It is indicated in patients with contraindication to fibrinolysis or when risk stratification in hemodynamically stable patients suggests increased morbidity and mortality [10].

Treatment of VTE depends on the long-term risk of recurrence after discontinuing anticoagulation; the duration varies between provoked VTE versus unprovoked VTE. Patients with provoked PE or with transient risk factors, such as major surgeries, immobilization, prolonged air travel, direct trauma to the leg, or hormone contraception, can be treated for three months based on the fact that the recurrence risk is $1 \%$ in the first year and $0.5 \%$ annually thereafter. On the other hand, patients with persistent risk factors or unprovoked PE should be treated indefinitely, with regular assessment of bleeding risk and anticoagulation 
benefit [11].

Recently, the use of warfarin has significantly reduced with increasing physician and patient preference for DOACs. Apixaban is an oral factor Xa inhibitor; it is a drug with rapid onset of action and predictable pharmacokinetics that allow a fixed-dose regimen. With these distinguishing properties, apixaban became a convenient and alternative to conventional therapy with vitamin $\mathrm{K}$ analog (warfarin) and subcutaneous low molecular weight heparin (enoxaparin). This was enforced by the AMPLIFY study [4], which showed noninferiority to warfarin and significantly decreased bleeding risk in the treatment of acute deep vein thrombosis.

Our patient had saddle pulmonary embolus while on the appropriate dose of anticoagulation with apixaban, which constitutes treatment failure. A decision was made to treat the patient with heparin infusion due to the urgent need for tight monitoring of drug levels with readily available laboratory assays (activated partial thromboplastin time) to ensure the therapeutic goal. Interventional radiology was consulted but the patient did not qualify for thrombolytic treatment.

Treatment failure of thromboembolism was previously mentioned in the literature, with an incidence rate of $2 \%$ [12-13]. One case report published in 2016, of a patient treated with different classes of DOACs, failed to resolve pulmonary clots who needed final treatment with warfarin [14]. Another case was reported in 2017 with recurrence of DVT while on apixaban. Although the absence of clinically available assays to evaluate medication plasma level and activity is not always associated with treatment failure, it was proposed by researchers [15]. Several attempts were made to correlate the effect of DOACs on coagulation laboratory studies, but the results are variable and need more understanding for validation [16].

In the Aristotle trial [17], apixaban plasma levels were measured using the liquid chromatography/mass spectrometry (HPLC/MS-MS) method, as well as the chromogenic anti-Xa method. Laboratory measurements detecting anti-Xa activity were considered useful for the quantification of drug levels for apixaban and rivaroxaban. It was shown that undetected anti-Xa activity excludes significant drug levels.

In comparison with other DOACs, the acute DVT [18] study, part of the EINSTEIN program, showed that recurrent venous thromboembolism (fatal pulmonary embolism, nonfatal pulmonary embolism, and recurrent deep venous thrombosis) all occurred while following patients being treated with rivaroxaban as well. Further management varies but considering a medication that can be tightly monitored for adequate drug levels is a valid alternative [15]. According to a single-center review [13] of 59 patients who failed treatment with DOACs, it was a common practice to switch to therapeutic low molecular weight heparin (LMWH) followed by another DOAC after successful parenteral therapy.

Increasing the dose of DOAC is another approach; in the RENOVATE clinical trial [12], a comparison between dabigatran etexilate $220 \mathrm{mg}$ and dabigatran etexilate $150 \mathrm{mg}$ showed that the composite of total VTE (symptomatic and venographic), in addition to the mortality rate, was $6 \%$ and $8.5 \%$, respectively, suggesting a dose-dependent decrease in the incidence of VTE while receiving dabigatran. On the other hand, patients who developed VTE while receiving rivaroxaban did not benefit from dose escalation [19]. This might indicate that increasing the dose is more effective before developing treatment failure.

A metanalysis of reported cases of treatment failure on DOACs observed that patients who had recurrent VTE had an underlying antiphospholipid syndrome, atrial fibrillation, or DVT [20]. Thus, the mechanism of failure may be in part due to an underlying condition, insufficient dose, or the different pharmacokinetics and pharmacodynamics of each DOAC [20].

It is certain that the risk of VTE recurrence is decreased with apixaban when compared with placebo, but for a subset of the population being treated with Apixaban, the recurrence of VTE or VTE-related death was $2.3 \%$ and the mortality rate was $1.5 \%$. The reported causes of death were PE, PE not ruled out, cancer, cardiovascular disease, and infectious causes [4]. Young adults have high mortality as well, and it has been increasing in comparison to the past decade [2]. Therefore, it is of paramount importance to diagnose recurrent VTE because any delay in detection and treatment would significantly increase mortality.

\section{Conclusions}

The emerging cases of recurrent VTE raise suspicion for the efficacy of DOACs. This challenges the concept of the patients' safety while on anticoagulants, promoting awareness about the condition. Such treatment failure should always be on the differential diagnosis of patients presenting with chest pain and shortness of breath even if they are appropriately anti-coagulated. In the future, perhaps, a clinically available test for the activity of DOACs would help decrease the number of similar cases.

\section{Additional Information}

\section{Disclosures}

Human subjects: Consent was obtained or waived by all participants in this study. Conflicts of interest: In 
compliance with the ICMJE uniform disclosure form, all authors declare the following: Payment/services info: All authors have declared that no financial support was received from any organization for the submitted work. Financial relationships: All authors have declared that they have no financial relationships at present or within the previous three years with any organizations that might have an interest in the submitted work. Other relationships: All authors have declared that there are no other relationships or activities that could appear to have influenced the submitted work.

\section{References}

1. Badireddy M, Mudipalli VR: Deep Venous Thrombosis Prophylaxis. StatPearls [Internet], Treasure Island (FL); 2021.

2. Martin KA, Molsberry R, Cuttica MJ, Desai KR, Schimmel DR, Khan SS: Time Trends in Pulmonary Embolism Mortality Rates in the United States, 1999 to 2018. J Am Heart Assoc. 2020, 9:e016784. 10.1161/JAHA.120.016784

3. Mazzolai L, Aboyans V, Ageno W, et al.: Diagnosis and management of acute deep vein thrombosis: a joint consensus document from the European Society of Cardiology working groups of aorta and peripheral vascular diseases and pulmonary circulation and right ventricular function. Eur Heart J. 2018, 39:4208-1. 10.1093/eurheartj/ehx003

4. Agnelli G, Buller HR, Cohen A, et al.: Oral apixaban for the treatment of acute venous thromboembolism . N Engl J Med. 2013, 369:799-808. 10.1056/NEJMoa1302507

5. Schulman S, Kakkar AK, Goldhaber SZ, et al.: Treatment of acute venous thromboembolism with dabigatran or warfarin and pooled analysis. Circulation. 2014, 129:764-72. 10.1161/CIRCULATIONAHA.113.004450

6. Haines DE, Raabe DS, Gundel WD, Wackers FJ: Anatomic and prognostic significance of new T-wave inversion in unstable angina. Am J Cardiol. 1983, 52:14-8. 10.1016/0002-9149(83)90061-9

7. Giordano NJ, Jansson PS, Young MN, Hagan KA, Kabrhel C: Epidemiology, pathophysiology, stratification, and natural history of pulmonary embolism. Tech Vasc Interv Radiol. 2017, 20:135-40. 10.1053/j.tvir.2017.07.002

8. Jaff MR, McMurtry MS, Archer SL, et al.: Management of massive and submassive pulmonary embolism, iliofemoral deep vein thrombosis, and chronic thromboembolic pulmonary hypertension: a scientific statement from the American Heart Association. Circulation. 2011, 123:1788-830.

10.1161/CIR.0b013e318214914f

9. Nelson SM, Craig SH: Ekosonic Endovascular System (EKOS) in a trauma patient with intracranial bleed, recent major surgery, and massive pulmonary embolus: a case report. Radiol Case Rep. 2018, 13:156-60. 10.1016/j.radcr.2017.10.005

10. Engelberger RP, Kucher N: Catheter-based reperfusion treatment of pulmonary embolism. Circulation. 2011, 124:2139-44. 10.1161/CIRCULATIONAHA.111.023689

11. Kearon C, Akl EA: Duration of anticoagulant therapy for deep vein thrombosis and pulmonary embolism Blood. 2014, 123:1794-801. 10.1182/blood-2013-12-512681

12. Eriksson BI, Dahl OE, Rosencher N, et al.: Dabigatran etexilate versus enoxaparin for prevention of venous thromboembolism after total hip replacement: a randomised, double-blind, non-inferiority trial. Lancet. 2007, 370:949-56. 10.1016/S0140-6736(07)61445-7

13. McIlroy G, Smith N, Lokare A, Beale K, Kartsios C: Treatment failure in patients receiving direct oral anticoagulants: clinical management and outcomes from a single-center review of 59 consecutive patients. Blood. 2018, 132:5058. 10.1182/blood-2018-99-111108

14. Rankin J, Nagar M, Crosby J, Toomari N, Pietras R, Ben-Zur UM: Possible failure of novel direct-acting oral anticoagulants in management of pulmonary embolism: a case report. J Med Case Rep. 2016, 10:346. 10.1186/s13256-016-1135-9

15. Sharma M, Ramanathan S, Khalighi K: Development of new deep venous thrombosis while on apixaban . Case Rep Cardiol. 2017, 2017:2842935. 10.1155/2017/2842935

16. Douxfils J, Ageno W, Samama CM, et al.: Laboratory testing in patients treated with direct oral anticoagulants: a practical guide for clinicians. J Thromb Haemost. 2018, 16:209-19. 10.1111/jth.13912

17. Cuker A, Siegal DM, Crowther MA, Garcia DA: Laboratory measurement of the anticoagulant activity of the non-vitamin K oral anticoagulants. J Am Coll Cardiol. 2014, 64:1128-39. 10.1016/j.jacc.2014.05.065

18. Bauersachs R, Berkowitz SD, Brenner B, et al.: Oral rivaroxaban for symptomatic venous thromboembolism. N Engl J Med. 2010, 363:2499-510. 10.1056/NEJMoa1007903

19. Eriksson BI, Borris LC, Dahl OE, et al.: Dose-escalation study of rivaroxaban (BAY 59-7939) - an oral, direct Factor Xa inhibitor - for the prevention of venous thromboembolism in patients undergoing total hip replacement. Thromb Res. 2007, 120:685-93. 10.1016/j.thromres.2006.12.025

20. Kajy M, Mathew A, Ramappa P: Treatment failures of direct oral anticoagulants . Am J Ther. 2021, 28:e87-95. 10.1097/MJT.0000000000001083 